Forum 2018 · 33:206

https://doi.org/10.1007/s12312-018-0432-1

Online publiziert: 3. Mai 2018

C) Springer Medizin Verlag $\mathrm{GmbH}$, ein Teil von

Springer Nature 2018

\section{Schleswig-Holsteinische \\ Krebsgesellschaft e.V.}

Schleswig Holsteinische Krebsgesellschaft e.V.

Kiel, Deutschland

\title{
Erstmals in Kiel: Seminarreihe für Angehörige von Krebspatienten
}

so stark sein müssten. Um den Erkrankten zu schonen, halten sie ihre Gefühle zurück oder verlieren eigene Bedürfnisse aus dem Blick. Das kann schnell überfordern.

\section{》) Angehörige halten oft eigene Bedürfnisse zurück}

In Kiel lud die Schleswig-Holsteinische Krebsgesellschaft im Februar deshalb gemeinsam mit der Selbsthilfekontaktstelle KIBIS zu einer Seminarreihe für Angehörige von Krebspatienten ein. Themen des Gruppenangebotes waren u.a.: Wie wir miteinander reden können, Entspannung, Sozialrecht und Palliativmedizin. Darüber hinaus gab es immer wieder Zeit für den Austausch untereinander.

Am Ende des Kurses standen viele neue Erkenntnisse und positive Rückmeldungen. Die Teilnehmer lernten den Austausch so sehr schätzen, dass sie sich als Selbsthilfegruppe für Angehörige von krebskranken Menschen weiterhin monatlich in Kiel treffen.

\section{Korrespondenzadresse}

\section{Sonja Kulschewski}

Schleswig-Holsteinische Krebsgesellschaft e.V. Alter Markt 1-2, 24103 Kiel, Deutschland info@krebsgesellschaft-sh.de www.krebsgesellschaft-sh.de wältigen?", so Papke weiter. Denn viele Angehörige glauben, dass sie jetzt doppelt

\section{Muskuloskelettale Tumore}

Lesen Sie: Der Onkologe

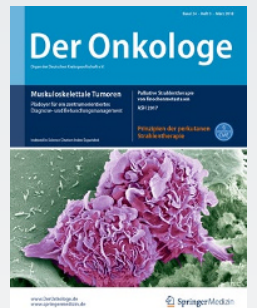

Muskuloskelettale Tumore ist das Leitthema in Ausgabe 3/18 von DerOnkologe.

Diese Tumoren treten als Weichteilsarkome oder Knochensarkome auf. Die Inzidenz ist selten, das Kollektiv sehr inhomogen. Eine interdisziplinäre Behandlung an spezialisierten Zentren ist somit empfehlenswert und wird praktiziert. Sie führte in den letzten Jahrzehnten zu einem Fortschritt in Diagnostik und Therapie.
Im aktuellen Heft von Der Onkologe wird das Thema aus den unterschiedlichen Fachrichtungen beleuchtet. Unter anderem werden die Weiterentwicklungen und die Verfeinerung der histopathologischen und molekulargenetischen Diagnostik beschrieben. Der integrative Bestandteil der Strahlentherapie und die aktuellen medikamentösen Konzepte und neuen Substanzen in der interdisziplinären Behandlung von Sarkomen sind weitere Themen.

www.DerOnkologe.de 